\title{
A NEW ERROR ANALYSIS FOR DISCONTINUOUS FINITE ELEMENT METHODS FOR LINEAR ELLIPTIC PROBLEMS
}

\author{
THIRUPATHI GUDI
}

\begin{abstract}
The standard a priori error analysis of discontinuous Galerkin methods requires additional regularity on the solution of the elliptic boundary value problem in order to justify the Galerkin orthogonality and to handle the normal derivative on element interfaces that appear in the discrete energy norm. In this paper, a new error analysis of discontinuous Galerkin methods is developed using only the $H^{k}$ weak formulation of a boundary value problem of order $2 k$. This is accomplished by replacing the Galerkin orthogonality with estimates borrowed from a posteriori error analysis and by using a discrete energy norm that is well defined for functions in $H^{k}$.
\end{abstract}

\section{INTRODUCTION}

Let $\Omega \subset \mathbb{R}^{n}$, be a bounded polygonal domain and $f \in L_{2}(\Omega)$. For simplicity, we assume $n=2$. However, our error analysis may be extended to any $n \geq 1$. To give the motivation, consider a model problem of finding $u \in H_{0}^{1}(\Omega)$ such that

$$
\int_{\Omega} \nabla u \cdot \nabla v d x=\int_{\Omega} f v d x \forall v \in H_{0}^{1}(\Omega) .
$$

Many discontinuous Galerkin (DG) methods 3 have been proposed for (1.1) based on a discrete space

$$
V_{h} \subseteq\left\{v \in L_{2}(\Omega):\left.v\right|_{T} \in P_{r}(T) \forall T \in \mathcal{T}_{h}\right\},
$$

where $\mathcal{T}_{h}$ is a triangulation of the computational domain $\Omega$. For these methods, a priori error estimates are derived based on Galerkin orthogonality and Cea's Lemma assuming that the solution $u$ of (1.1) has the following regularity:

$$
\left.u\right|_{T} \in H^{s}(T), \forall T \in \mathcal{T}_{h}, s>\frac{3}{2} .
$$

To be more precise, consider the variational form of the symmetric interior penalty method [22, 39, 2] : Find $u_{h} \in V_{h}$ such that

$$
a_{h}\left(u_{h}, v_{h}\right)=\int_{\Omega} f v_{h} d x \forall v_{h} \in V_{h},
$$

Received by the editor January 5, 2009 and, in revised form, June 16, 2009

2010 Mathematics Subject Classification. Primary 65N30, 65N15.

Key words and phrases. Optimal error estimates, elliptic regularity, finite element, discontinuous Galerkin, nonconforming.

(C)2010 American Mathematical Society Reverts to public domain 28 years from publication 
where

$$
\begin{gathered}
a_{h}(w, v)=\sum_{T \in \mathcal{T}_{h}} \int_{T} \nabla w \cdot \nabla v d x-\sum_{e \in \mathcal{E}_{h}} \int_{e}(\{\{\nabla w\}\} \llbracket v \rrbracket+\{\{\nabla v\} \rrbracket \llbracket w \rrbracket d s \\
\quad+\sum_{e \in \mathcal{E}_{h}} \int_{e} \frac{\sigma}{h_{e}} \llbracket w \rrbracket \llbracket v \rrbracket d s \quad w, v \in V_{h},
\end{gathered}
$$

the jumps and means are defined as in Section 3 and $\sigma$ is a sufficiently large positive constant.

By writing the solution $u$ of (1.1) as the sum of a regular part and a singular part [27, the following integration by parts formula [15, Lemma 2.1] can be proved under the regularity result that the solution $u \in H^{s}(\Omega)$ for $s>3 / 2$ [27]:

$$
\int_{T} \nabla u \cdot \nabla v d x=\int_{\partial T} \nabla u \cdot v n d s+\int_{T} f v d x \quad \forall T \in \mathcal{T}_{h}, \forall v \in V_{h} .
$$

Hence the solution $u$ of (1.1) satisfies

$$
a_{h}\left(u, v_{h}\right)=\int_{\Omega} f v_{h} d x \quad \forall v_{h} \in V_{h},
$$

which implies the following Galerkin orthogonality:

$$
a_{h}\left(u-u_{h}, v_{h}\right)=0 \quad \forall v_{h} \in V_{h} .
$$

Then the following a priori error estimate [35, 15, 13, 14, 34, is obtained from (1.3):

$$
\left\|u-u_{h}\right\|_{1, h} \leq C \inf _{v_{h} \in V_{h}}\left\|u-v_{h}\right\|_{1, h}
$$

where for $w \in H^{s}(\Omega)+V_{h}, s>3 / 2$,

$$
\|w\|_{1, h}^{2}=\sum_{T \in \mathcal{T}_{h}}\|\nabla w\|_{L_{2}(T)}^{2}+\sum_{e \in \mathcal{E}_{h}} h_{e}\|\{\{\nabla w\}\}\|_{L_{2}(e)}^{2}+\sum_{e \in \mathcal{E}_{h}} \frac{\sigma}{h_{e}}\|\llbracket w \rrbracket\|_{L_{2}(e)}^{2}
$$

and $h_{e}$ is the length of $e$. Note that the regularity result $u \in H^{s}(\Omega)$ for $s>3 / 2$ is needed to handle the term $\left\|\left\{\left\{\nabla\left(u-v_{h}\right)\right\}\right\}\right\|_{L_{2}(e)}$ in (1.4).

We see from the discussion above that the derivation of (1.3) for discontinuous Galerkin methods requires the nontrivial elliptic regularity theory in polygonal domains. The goal of this paper is to provide a new type of error estimates that does not require such regularity results. This new approach is particularly useful for more complicated problems, such as linear interface, where $u$ has low regularity 31.

We will derive the new error estimates by an analog of the Berger-Scott-Strang lemma [5] that decomposes the error into two parts in which one measures the interpolation error and the other measures the nonconforming error and the consistency error together. Thereby the analysis does not require any regularity other than that the weak solution of a PDE of order $2 k$ belongs to $H^{k}(\Omega)$. We obtain the following error estimate for (1.2):

$$
\left\|u-u_{h}\right\|_{h} \leq C\left(\inf _{v_{h} \in V_{h}}\left\|u-v_{h}\right\|_{h}+O s c_{k}(f)\right),
$$

where

$$
\|w\|_{h}^{2}=\sum_{T \in \mathcal{T}_{h}}\|\nabla w\|_{L_{2}(T)}^{2}+\sum_{e \in \mathcal{E}_{h}} \frac{\sigma}{h_{e}}\|\llbracket w \rrbracket\|_{L_{2}(e)}^{2} \quad w \in H^{1}(\Omega)+V_{h}
$$


and $O s c_{k}(f)$ which measures the oscillations of $f$ is defined in (2.8). Note on the right-hand sides of (1.5) that the first term quantifies the interpolation error and the second term measures the oscillations of $f$ which are of the same order with the first term (assuming that $u$ has enough regularity) if $f \in L_{2}(\Omega)$ and higher order if $f$ is sufficiently smooth. Our error analysis is motivated by the recent a posteriori error analysis for discontinuous Galerkin methods [16, 18, 30, 29] and the results of [8]. The key ingredients are the discrete local efficiency arguments 37, 38, for a posteriori error estimators and an enriching map for piecewise smooth functions 6. 7, 8, 9, 10.

The rest of the article is organized as follows. In Section 2, we present the main result in an abstract lemma which enables us to decompose the error. Section 3 and Section 4 are devoted to the applications of the abstract lemma to various nonconforming and discontinuous Galerkin methods for second and fourth order elliptic problems, respectively. Finally, in Section 5, we present conclusions and possible extensions.

\section{Abstract result}

Recall the Sobolev-Hilbert space $H^{k}(\Omega)$ which is the set of all $L_{2}(\Omega)$ functions whose distributional derivatives up to order $k$ are in $L_{2}(\Omega)$. Denote by $V:=H_{0}^{k}(\Omega)$, the set of all functions in $H^{k}(\Omega)$ whose traces up to order $k-1$ vanishes. Denote the norm on $V$ by $\|\cdot\|_{V}$. The model problem is to find $u \in V$ such that

$$
a(u, v)=(f, v) \quad \forall v \in V
$$

where $a(\cdot, \cdot)$ is the bilinear from for the underlying PDE of order $2 k$ and $(\cdot, \cdot)$ denotes the $L_{2}$ inner product. We assume that the bilinear form $a$ is bounded and elliptic so that the model problem (2.1) has a unique solution $u \in V$. Denote by $\mathcal{T}_{h}$ a regular (without hanging nodes) simplicial triangulation of $\Omega$. Let $h_{T}=\operatorname{diam} T$ and $h=\max \left\{h_{T}: T \in \mathcal{T}_{h}\right\}$. Let the discontinuous finite element space $V_{h}$ be a subspace of

$$
V_{h}^{r}=\left\{v_{h} \in L_{2}(\Omega):\left.v_{h}\right|_{T} \in P_{r}(T) \forall T \in \mathcal{T}_{h}\right\}
$$

where $\mathbb{P}_{r}(D)$ is the space of polynomials of degree less than or equal to $r$ restricted to the set $D$. Let $\|\cdot\|_{h}$ be a mesh dependent norm on $V+V_{h}$ and $V_{c}$ a finite element subspace of $V$ associated with $\mathcal{T}_{h}$.

The discontinuous finite element method is to find $u_{h} \in V_{h}$ such that

$$
a_{h}\left(u_{h}, v\right)=(f, v) \quad \forall v \in V_{h},
$$

where the bilinear form $a_{h}(\cdot, \cdot)$ is defined on $V_{h} \times\left(V_{c}+V_{h}\right)$.

We make the following abstract assumptions:

(N1) There is a positive constant $C$ independent of $h$ such that

$$
C\|v\|_{h}^{2} \leq a_{h}(v, v) \quad \forall v \in V_{h} .
$$

(N2) There is a positive constant $C$ independent of $h$ such that for all $w \in V_{c}$,

(N3) There exists a linear map $E_{h}: V_{h} \rightarrow V_{c}$ satisfying

$$
\left\|E_{h} v\right\|_{V} \leq C\|v\|_{h} \quad \forall v \in V_{h}
$$

for some positive constant $C$ independent of $h$. 
It can be readily seen from (2.3) that there is a unique solution $u_{h} \in V_{h}$ for (2.2). We are now ready to prove an abstract lemma.

Lemma 2.1. Let $u \in V:=H_{0}^{k}(\Omega)$ and $u_{h} \in V_{h}$ be the solutions of (2.1) and (2.2), respectively. Assume that the assumptions (N1)-(N3) hold. Then there exists a positive constant $C$ independent of $h$ such that

$$
\left\|u-u_{h}\right\|_{h} \leq C \inf _{v \in V_{h}}\left[\|u-v\|_{h}+\sup _{\phi \in V_{h} \backslash\{0\}} \frac{\left(f, \phi-E_{h} \phi\right)-a_{h}\left(v, \phi-E_{h} \phi\right)}{\|\phi\|_{h}}\right] .
$$

In addition, if there exists a positive constant $C$ independent of $h$ such that for any $v \in V_{h}$,

$$
\sup _{\phi \in V_{h} \backslash\{0\}} \frac{\left(f, \phi-E_{h} \phi\right)-a_{h}\left(v, \phi-E_{h} \phi\right)}{\|\phi\|_{h}} \leq C\left(\|u-v\|_{h}+O s c_{k}(f)\right),
$$

where

$$
O s c_{k}(f)=\left(\sum_{T \in \mathcal{T}_{h}} h_{T}^{2 k}\left[\inf _{\bar{f} \in P_{r-k}(T)}\|f-\bar{f}\|_{L_{2}(T)}^{2}\right]\right)^{1 / 2}
$$

then

$$
\left\|u-u_{h}\right\|_{h} \leq \tilde{C}\left(\inf _{v \in V_{h}}\|u-v\|_{h}+O s c_{k}(f)\right) .
$$

Here $\tilde{C}$ is a positive constant independent of $h$.

Proof. Let $v \in V_{h}$ be such that $v \neq u_{h}$. Let $\psi=u_{h}-v$. From (2.3), (2.2) and (2.1), we get

$$
\begin{aligned}
C\left\|u_{h}-v\right\|_{h}^{2} & \leq a_{h}\left(u_{h}-v, \psi\right) \\
& =(f, \psi)-a_{h}(v, \psi) \\
& =a\left(u, E_{h} \psi\right)-a_{h}\left(v, E_{h} \psi\right)+\left(f, \psi-E_{h} \psi\right)-a_{h}\left(v, \psi-E_{h} \psi\right) .
\end{aligned}
$$

We obtain

$$
\left\|u_{h}-v\right\|_{h} \leq C\left(\frac{a\left(u, E_{h} \psi\right)-a_{h}\left(v, E_{h} \psi\right)}{\left\|u_{h}-v\right\|_{h}}+\frac{\left(f, \psi-E_{h} \psi\right)-a_{h}\left(v, \psi-E_{h} \psi\right)}{\left\|u_{h}-v\right\|_{h}}\right) .
$$

Using (2.4) and (2.5), we find

$$
\begin{aligned}
\left|a\left(u, E_{h} \psi\right)-a_{h}\left(v, E_{h} \psi\right)\right| & \leq C\|u-v\|_{h}\left\|E_{h} \psi\right\|_{V} \\
& \leq C\|u-v\|_{h}\|\psi\|_{h} \\
& =C\|u-v\|_{h}\left\|u_{h}-v\right\|_{h},
\end{aligned}
$$

which implies that

$$
\frac{\left|a\left(u, E_{h} \psi\right)-a_{h}\left(v, E_{h} \psi\right)\right|}{\left\|u_{h}-v\right\|_{h}} \leq C\|u-v\|_{h} .
$$

It is obvious that

$$
\frac{\left(f, \psi-E_{h} \psi\right)-a_{h}\left(v, \psi-E_{h} \psi\right)}{\|\psi\|_{h}} \leq \sup _{\phi \in V_{h} \backslash\{0\}} \frac{\left(f, \phi-E_{h} \phi\right)-a_{h}\left(v, \phi-E_{h} \phi\right)}{\|\phi\|_{h}} .
$$

Now a use of the triangle inequality yields the estimate (2.6). Finally, we use (2.7) in (2.6) to complete the proof.

We have immediately the following corollary. 
Corollary 2.2. Assume that the hypothesis for Lemma 2.1 is true. Furthermore, assume that the oscillation $\mathrm{Osc}_{k}(f)$ is zero. Then, the error estimate (2.9) is quasioptimal in the sense that there is a positive constant $C$ independent of $h$ such that

$$
\left\|u-u_{h}\right\|_{h} \leq C \inf _{v \in V_{h}}\|u-v\|_{h} .
$$

\section{SEcond order problems $(k=1)$}

Here we have $V=H_{0}^{1}(\Omega)$ and $\|v\|_{V}=\|\nabla v\|_{L_{2}(\Omega)}$. For given $f \in L_{2}(\Omega)$, the model problem is to find $u \in V$ such that

$$
a(u, v)=(f, v) \quad \forall v \in V,
$$

where

$$
a(u, v)=\int_{\Omega} \nabla u \cdot \nabla v d x .
$$

We now introduce some notation. Denote the set of all interior edges of $\mathcal{T}_{h}$ by $\mathcal{E}_{h}^{i}$, the set of boundary edges by $\mathcal{E}_{h}^{b}$, and define $\mathcal{E}_{h}=\mathcal{E}_{h}^{i} \cup \mathcal{E}_{h}^{b}$. The length of any edge $e \in \mathcal{E}_{h}$ will be denoted by $h_{e}$. Define a broken Sobolev space

$$
H^{1}\left(\Omega, \mathcal{T}_{h}\right)=\left\{v \in L_{2}(\Omega): v_{T}=\left.v\right|_{T} \in H^{1}(T) \quad \forall T \in \mathcal{T}_{h}\right\} .
$$

For any $e \in \mathcal{E}_{h}^{i}$, there are two triangles $T_{+}$and $T_{-}$such that $e=\partial T_{+} \cap \partial T_{-}$. Let $n_{-}$be the unit normal of $e$ pointing from $T_{-}$to $T_{+}$, and $n_{+}=-n_{-}$. (cf. Fig. 3.1). For any $v \in H^{1}\left(\Omega, \mathcal{T}_{h}\right)$, we define the jump and mean of $v$ on $e$ by

$$
\llbracket v \rrbracket=v_{-} n_{-}+v_{+} n_{+} \text {and }\{\{v\}\}=\frac{1}{2}\left(v_{-}+v_{+}\right) \text {, respectively, }
$$

where $v_{ \pm}=\left.v\right|_{T_{ \pm}}$. Similarly, define for $w \in H^{1}\left(\Omega, \mathcal{T}_{h}\right)^{2}$ the jump and mean of $w$ on $e \in \mathcal{E}_{h}^{i}$ by

$$
\llbracket w \rrbracket=w_{-} \cdot n_{-}+w_{+} \cdot n_{+}, \text {and }\{\{w\}\}=\frac{1}{2}\left(w_{-}+w_{+}\right), \text {respectively, }
$$

where $w_{ \pm}=\left.w\right|_{T_{ \pm}}$.

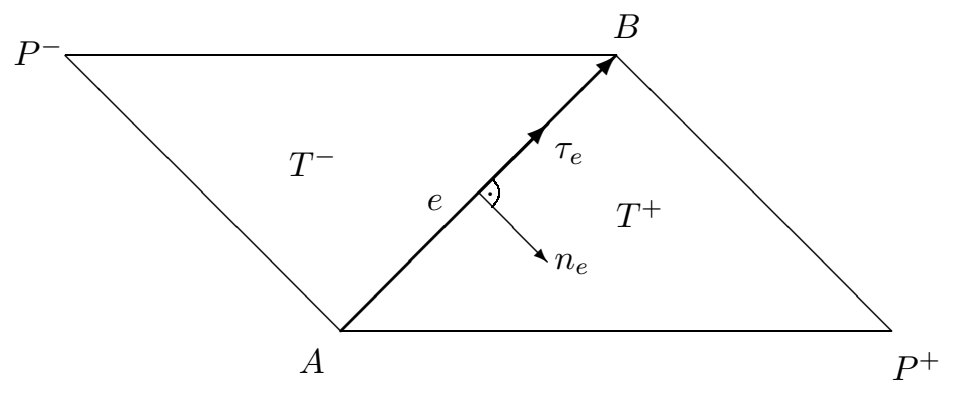

Figure 3.1. Two neighboring triangles $T_{-}$and $T_{+}$that share the edge $e=\partial T_{-} \cap \partial T_{+}$with initial node $A$ and end node $B$ and unit normal $n_{e}$. The orientation of $n_{e}=n_{-}=-n_{+}$equals the outer normal of $T_{-}$, and hence, points into $T_{+}$. 
For any edge $e \in \mathcal{E}_{h}^{b}$, there is a triangle $T \in \mathcal{T}_{h}$ such that $e=\partial T \cap \partial \Omega$. Let $n_{e}$ be the unit normal of $e$ that points outside $T$. For any $v \in H^{1}(T)$, we set on $e \in \mathcal{E}_{h}^{b}$,

$$
\llbracket v \rrbracket=v n_{e} \text { and }\{\{v\}\}=v,
$$

and for $w \in H^{1}(T)^{2}$,

$$
\llbracket w \rrbracket=w \cdot n_{e} \text {, and }\{\{w\}\}=w .
$$

We recall the following trace inequality on $V_{h}$ [11, 19.

Lemma 3.1. There exists a positive $C$ independent of $h$ such that for $v_{h} \in V_{h}^{r}$,

$$
\left\|v_{h}\right\|_{L_{2}(e)} \leq C h_{e}^{-1 / 2}\left\|v_{h}\right\|_{L_{2}(T)} \forall T \in \mathcal{T}_{h},
$$

where $e$ is an edge of $T$.

Throughout this section, $\Delta$ denotes the Laplacian.

The proof of the following lemma is similar to well-known discrete local efficiency estimates [1, 38, 37, 30 in a posteriori error analysis of second order problems when $v=u_{h}$ is the finite element solution. We state the result here and omit the proof.

Lemma 3.2. Let $v \in V_{h}$. Then there is a positive constant $C$ independent of $h$ such that

$$
\sum_{T \in \mathcal{T}_{h}} h_{T}^{2}\|f+\Delta v\|_{L_{2}(T)}^{2} \leq C\left(\sum_{T \in \mathcal{T}_{h}}\|\nabla(u-v)\|_{L_{2}(T)}^{2}+O s c_{1}(f)^{2}\right)
$$

and

$$
\sum_{e \in \mathcal{E}_{h}^{i}} h_{e}\|\llbracket \nabla v \rrbracket\|_{L_{2}(e)}^{2} \leq C\left(\sum_{T \in \mathcal{T}_{h}}\|\nabla(u-v)\|_{L_{2}(T)}^{2}+O s c_{1}(f)^{2}\right),
$$

where $O s c_{1}(f)$ is defined in (2.8).

Let $V_{c}=V_{h} \cap V$ be the conforming finite element space. The construction of an enriching map $E_{h}: V_{h} \rightarrow V_{c}$ can be done by averaging [6, 7, 8]. Let $p$ be any interior node of the Lagrange $P_{r}$ finite element space associated with the triangulation $\mathcal{T}_{h}$ and let $\mathcal{T}_{p}$ be the set of all triangles sharing the node $p$. For $v \in V_{h}$, define $E_{h} v \in V_{c}$ by

$$
E_{h} v(p)=\left.\frac{1}{\left|\mathcal{T}_{p}\right|} \sum_{T \in \mathcal{T}_{p}} v\right|_{T}(p)
$$

where $\left|\mathcal{T}_{p}\right|$ is the cardinality of $\mathcal{T}_{p}$. For all boundary nodes $p$, set $E_{h} v(p)=0$. For the rest of the section, we use this $E_{h}$.

We now present the applications of Lemma 2.1 to a wide range of discontinuous finite element methods.

3.1. Classical nonconforming method. The discrete space is the CrouzeixRaviart 21] nonconforming $P_{1}$ finite element space defined by

$$
V_{h}=\left\{v_{h} \in L_{2}(\Omega):\left.v_{h}\right|_{T} \in P_{1}(T) \forall T \in \mathcal{T}_{h}, \int_{e} \llbracket v \rrbracket d s=0 \forall e \in \mathcal{E}_{h}\right\} .
$$

Define the norm $\|\cdot\|_{h}$ by

$$
\|v\|_{h}^{2}=\sum_{T \in \mathcal{T}_{h}} \int_{T}|\nabla v|^{2} d x
$$


The nonconforming approximate solution $u_{h} \in V_{h}$ is obtained by solving

$$
a_{h}\left(u_{h}, v\right)=(f, v) \quad \forall v \in V_{h},
$$

where

$$
a_{h}(w, v)=\sum_{T \in \mathcal{T}_{h}} \int_{T} \nabla w \cdot \nabla v d x \quad \forall w, v \in V_{h} .
$$

It is easy to check the assumptions (N1) and (N2). The enriching map $E_{h}$ in (3.6) satisfies [6, 7, 8, 30]

$$
\sum_{T \in \mathcal{T}_{h}} h_{T}^{-2}\left\|E_{h} v-v\right\|_{L_{2}(T)}^{2}+\left\|E_{h} v\right\|_{V}^{2} \leq C\|v\|_{h}^{2} \quad \forall v \in V_{h} .
$$

Therefore, the estimate (2.6) is valid for the nonconforming method (3.9).

We now verify the estimate (2.7). For this, let $v, \phi \in V_{h}$ and denote $\psi=\phi-E_{h} \phi$. Using (3.3), (3.4), (3.5) and (3.11),

$$
\begin{aligned}
(f, \psi)-a_{h}(v, \psi)= & \left.(f, \psi)-\sum_{T \in \mathcal{T}_{h}} \int_{T} \nabla v \cdot \nabla \psi d x=(f, \psi)-\sum_{e \in \mathcal{E}_{h}^{i}} \int_{e} \llbracket \nabla v \rrbracket\{\psi\}\right\} d s \\
\leq & C \sum_{T \in \mathcal{T}_{h}}\|f\|_{L_{2}(T)}\left\|\phi-E_{h} \phi\right\|_{L_{2}(T)} \\
& +C \sum_{e \in \mathcal{E}_{h}^{i}}\|\llbracket \nabla v \rrbracket\|_{L_{2}(e)}\left\|\left\{\phi \phi-E_{h} \phi\right\}\right\|_{L_{2}(e)} \\
\leq & C\left(\sum_{T \in \mathcal{T}_{h}} h_{T}^{2}\|f\|_{L_{2}(T)}^{2}+\sum_{e \in \mathcal{E}_{h}^{i}} h_{e}\|\llbracket \nabla v \rrbracket\|_{L_{2}(e)}^{2}\right)^{1 / 2}\|\phi\|_{h} \\
\leq & C\left(\|u-v\|_{h}+O s c_{1}(f)\right)\|\phi\|_{h} .
\end{aligned}
$$

Therefore,

$$
\sup _{\phi \in V_{h} \backslash\{0\}} \frac{\left(f, \phi-E_{h} \phi\right)-a_{h}\left(v, \phi-E_{h} \phi\right)}{\|\phi\|_{h}} \leq C\left(\|u-v\|_{h}+O s c_{1}(f)\right) .
$$

Hence, the estimate (2.9) holds for the nonconforming finite element method.

3.2. Discontinuous Galerkin (DG) methods. In this section, we present the application of Lemma 2.1 to discontinuous Galerkin methods [3] for second order elliptic problems.

The DG finite element space is defined by

$$
V_{h}=\left\{v_{h} \in L_{2}(\Omega):\left.v_{h}\right|_{T} \in P_{r}(T) \forall T \in \mathcal{T}_{h}\right\}
$$

for any $r \geq 1$. Define the norm $\|\cdot\|_{h}$ on $V_{h}$ by

$$
\|v\|_{h}^{2}=\sum_{T \in \mathcal{T}_{h}} \int_{T}|\nabla v|^{2} d x+\sum_{e \in \mathcal{E}_{h}} \frac{\sigma}{h_{e}}\|\llbracket v \rrbracket\|_{L_{2}(e)}^{2},
$$

where $\sigma>0$ is the stabilizing parameter.

The enriching map $E_{h}$ in (3.6) satisfies [6, 7, 8, 30]

$$
\sum_{T \in \mathcal{T}_{h}} h_{T}^{-2}\left\|E_{h} v-v\right\|_{L_{2}(T)}^{2}+\left\|E_{h} v\right\|_{V}^{2} \leq C\|v\|_{h}^{2} \quad \forall v \in V_{h} .
$$

This validates the assumption (N3). 
In order to define DG methods, we introduce the following. Let $W_{h}=V_{h} \times V_{h}$. Given $e \in \mathcal{E}_{h}$, define the local lifting operators $r_{e}: L_{2}(e)^{2} \rightarrow W_{h}$ and $\ell_{e}: L_{2}(e) \rightarrow$ $W_{h}$ by

$$
\begin{aligned}
& \int_{\Omega} r_{e}(q) \cdot \tau_{h} d x=\int_{e} q \cdot\left\{\left\{\tau_{h}\right\}\right\} d s \quad \text { for all } \tau_{h} \in W_{h}, \\
& \int_{\Omega} \ell_{e}(v) \cdot \tau_{h} d x=\int_{e} v \llbracket \tau_{h} \rrbracket d s \quad \text { for all } \tau_{h} \in W_{h} .
\end{aligned}
$$

The global lifting operators $r: L_{2}\left(\mathcal{E}_{h}\right)^{2} \rightarrow W_{h}$ and $\ell: L_{2}\left(\mathcal{E}_{h}^{i}\right) \rightarrow W_{h}$,

$$
r:=\sum_{e \in \mathcal{E}_{h}} r_{e} \quad \text { and } \quad \ell:=\sum_{e \in \mathcal{E}_{h}^{i}} \ell_{e}
$$

satisfy

$$
\begin{aligned}
& \int_{\Omega} r(q) \cdot \tau_{h} d x=\sum_{e \in \mathcal{E}_{h}} \int_{e} q \cdot\left\{\left\{\tau_{h}\right\}\right\} d s \quad \text { for all } \tau_{h} \in W_{h}, \\
& \int_{\Omega} \ell(v) \cdot \tau_{h} d x=\sum_{e \in \mathcal{E}_{h}^{i}} \int_{e} v \cdot \llbracket r_{h} \rrbracket d s \quad \text { for all } \tau_{h} \in W_{h} .
\end{aligned}
$$

The variational form of the DG methods [17] is to find $u_{h} \in V_{h}$ such that

$$
a_{h}\left(u_{h}, v\right)=(f, v) \quad \forall v \in V_{h},
$$

where for $w, v \in V_{h}$,

$$
\begin{aligned}
a_{h}(w, v)= & \sum_{T \in \mathcal{T}_{h}} \int_{T} \nabla w \cdot \nabla v d x-\sum_{e \in \mathcal{E}_{h}} \int_{e}(\{\{\nabla w\}\} \llbracket v \rrbracket+\theta\{\{\nabla v\}\} \llbracket w \rrbracket) d s \\
& +\sum_{e \in \mathcal{E}_{h}^{i}} \int_{e}(\beta \cdot \llbracket w \rrbracket \llbracket \nabla v \rrbracket+\llbracket \nabla w \rrbracket \beta \cdot \llbracket v \rrbracket) d s \\
& +\int_{\Omega} \alpha[r(\llbracket w \rrbracket)+\ell(\beta \cdot \llbracket w \rrbracket)] \cdot[r(\llbracket v \rrbracket)+\ell(\beta \cdot \llbracket v \rrbracket)] d s \\
& +\sum_{e \in \mathcal{E}_{h}} \int_{e} \frac{\sigma}{h_{e}} \llbracket w \rrbracket \llbracket v \rrbracket d s
\end{aligned}
$$

for $\theta= \pm, \alpha \in\{0,1\}, \beta \in \mathbb{R}^{2}$ and $\sigma>0$. Here $\theta=1, \beta=(0,0)$ and $\alpha=0$ give the symmetric interior penalty method (SIPG) $22,39,2], \theta=-1, \beta=(0,0)$ and $\alpha=0$ give the nonsymmetric interior penalty method (NIPG) [36], and $\theta=1, \beta \in \mathbb{R}^{2}$ and $\alpha=1$ give the local discontinuous Galerkin method (LDG) [20, 17].

Under the assumption $\sigma \geq \sigma_{*}>0\left(\sigma_{*}\right.$ is sufficiently large for SIPG and $\sigma_{*}>0$ for NIPG and LDG), it is known 3 that there is a positive constant $C$ such that $C\|v\|_{h}^{2} \leq a_{h}(v, v)$ for all $v \in V_{h}$. This verifies the assumption (N1).

For $v \in V, w \in V_{c}$ and $v_{h} \in V_{h}$, note that

$$
\begin{aligned}
a(v, w)-a_{h}\left(v_{h}, w\right)= & \sum_{T \in \mathcal{T}_{h}} \int_{T} \nabla\left(v-v_{h}\right) \cdot \nabla w d x-\sum_{e \in \mathcal{E}_{h}} \int_{e}\{\{\nabla w\}\} \llbracket v-v_{h} \rrbracket d s \\
& +\sum_{e \in \mathcal{E}_{h}^{i}} \int_{e} \beta \cdot \llbracket \nabla w \rrbracket \llbracket v-v_{h} \rrbracket d s
\end{aligned}
$$


where we have used the fact that for all $e \in \mathcal{E}_{h}, \llbracket v \rrbracket=0$. A use of the trace inequality (3.3) implies that

$$
\begin{aligned}
\left|a(v, w)-a_{h}\left(v_{h}, w\right)\right| \leq & \sum_{T \in \mathcal{T}_{h}}\left\|\nabla\left(v-v_{h}\right)\right\|_{L^{2}(T)}\|\nabla w\|_{L^{2}(T)} \\
& \left.+\sum_{e \in \mathcal{E}_{h}} \|\{\nabla w\}\right\}\left\|_{L^{2}(e)}\right\| \llbracket v-v_{h} \rrbracket \|_{L^{2}(e)} \\
& +\sum_{e \in \mathcal{E}_{h}^{i}}\|\beta \cdot \llbracket \nabla w \rrbracket\|_{L^{2}(e)}\left\|\llbracket v-v_{h} \rrbracket\right\|_{L^{2}(e)} \\
\leq & \sum_{T \in \mathcal{T}_{h}}\left\|\nabla\left(v-v_{h}\right)\right\|_{L^{2}(T)}\|\nabla w\|_{L^{2}(T)} \\
& +C_{\sigma_{*}, \beta}\left(\sum_{T \in \mathcal{T}_{h}}\|\nabla w\|_{L^{2}(T)}^{2}\right)^{1 / 2}\left(\sum_{e \in \mathcal{E}_{h}} \frac{\sigma}{h_{e}}\left\|\llbracket v-v_{h} \rrbracket\right\|_{L^{2}(e)}^{2}\right)^{1 / 2} .
\end{aligned}
$$

Hence, the assumption (N2) holds.

We now verify the estimate (2.7). Let $v, \phi \in V_{h}$ and denote $\psi=\phi-E_{h} \phi$. Then

$$
\begin{aligned}
(f, \psi)-a_{h}(v, \psi)= & \sum_{T \in \mathcal{T}_{h}} \int_{T}(f+\Delta v) \psi d x-\sum_{e \in \mathcal{E}_{h}^{i}} \int_{e} \llbracket \nabla v \rrbracket\{\{\psi\} d s \\
& +\theta \sum_{e \in \mathcal{E}_{h}} \int_{e}\{\{\nabla \psi\}\} \llbracket v \rrbracket d s-\sum_{e \in \mathcal{E}_{h}} \int_{e} \frac{\sigma}{h_{e}} \llbracket v \rrbracket \llbracket \psi \rrbracket d s \\
& -\sum_{e \in \mathcal{E}_{h}^{i}} \int_{e} \alpha(\beta \cdot \llbracket v \rrbracket \llbracket \nabla \psi \rrbracket+\llbracket \nabla v \rrbracket \beta \cdot \llbracket \psi \rrbracket) d s \\
& -\int_{\Omega}[r(\llbracket v \rrbracket)+\ell(\beta \cdot \llbracket v \rrbracket)] \cdot[r(\llbracket \psi \rrbracket)+\ell(\beta \cdot \llbracket \psi \rrbracket)] d s .
\end{aligned}
$$

Using the bounds for $r$ and $\ell$ in 3 that

$$
\begin{gathered}
\|r(\llbracket v \rrbracket)\|_{L_{2}(\Omega)}^{2} \leq C \sum_{e \in \mathcal{E}_{h}} \frac{1}{h_{e}}\|\llbracket v \rrbracket\|_{L_{2}(e)}^{2} \quad \forall v \in V_{h}, \\
\|\ell(\beta \cdot \llbracket v \rrbracket)\|_{L_{2}(\Omega)}^{2} \leq C \sum_{e \in \mathcal{E}_{h}^{I}} \frac{1}{h_{e}}\|\llbracket v \rrbracket\|_{L_{2}(e)}^{2} \quad \forall v \in V_{h},
\end{gathered}
$$

the trace inequality (3.3), (3.12) and Lemma 3.2, we obtain

$$
\sup _{\phi \in V_{h} \backslash\{0\}} \frac{\left(f, \phi-E_{h} \phi\right)-a_{h}\left(v, \phi-E_{h} \phi\right)}{\|\phi\|_{h}} \leq C\left(\|u-v\|_{h}+O s c_{1}(f)\right)
$$

and hence we conclude the error estimate in (2.9) for the DG methods in (3.13).

3.3. Weakly over-penalized symmetric interior penalty (WOPSIP) method. Here we highlight that the above analysis is also applicable to overpenalized interior penalty methods. We consider the weakly over-penalized symmetric interior penalty method in [15]. For any $v \in H^{1}\left(\Omega, \mathcal{T}_{h}\right)$, define on $e \in \mathcal{E}_{h}$,

$$
\pi_{e}(\llbracket v \rrbracket)=\frac{1}{h_{e}} \int_{e} \llbracket v \rrbracket d s .
$$


Note that $\pi_{e}(\llbracket u \rrbracket)=0$ for all $e \in \mathcal{E}_{h}$. Define

$$
V_{h}=\left\{v_{h} \in L_{2}(\Omega):\left.v_{h}\right|_{T} \in P_{1}(T) \forall T \in \mathcal{T}_{h}\right\},
$$

and the norm $\|\cdot\|_{h}$ on $V_{h}$ by

$$
\|v\|_{h}^{2}=\sum_{T \in \mathcal{T}_{h}} \int_{T}|\nabla v|^{2} d x+\sum_{e \in \mathcal{E}_{h}} \frac{1}{h_{e}^{2}} \pi_{e}(\llbracket v \rrbracket)^{2} .
$$

The WOPSIP method is to find $u_{h} \in V_{h}$ such that

$$
a_{h}\left(u_{h}, v\right)=(f, v) \quad \forall v \in V_{h},
$$

where for $w, v \in V_{h}$,

$$
a_{h}(w, v)=\sum_{T \in \mathcal{T}_{h}} \int_{T} \nabla w \cdot \nabla v d x+\sum_{e \in \mathcal{E}_{h}} \frac{1}{h_{e}^{2}} \pi_{e}(\llbracket w \rrbracket) \pi_{e}(\llbracket v \rrbracket) .
$$

Let $E_{h}: V_{h} \rightarrow V_{c}$ be defined as in (3.6), where $V_{c}$ is the conforming $P_{1}$ finite element space. Then it can be seen [6, 7, 8, that

$$
\sum_{T \in \mathcal{T}_{h}} h_{T}^{-2}\left\|E_{h} v-v\right\|_{L_{2}(T)}^{2}+\left\|E_{h} v\right\|_{V}^{2} \leq C\|v\|_{h}^{2} \quad \forall v \in V_{h} .
$$

Note that

$$
a_{h}(v, v)=\|v\|_{h}^{2} \quad \forall v \in V_{h}
$$

and for $v \in V, w \in V_{c}$ and $v_{h} \in V_{h}$,

$$
\left|a(v, w)-a_{h}\left(v_{h}, w\right)\right|=\sum_{T \in \mathcal{T}_{h}} \int_{T} \nabla\left(v-v_{h}\right) \cdot \nabla w d x \leq C\left\|v-v_{h}\right\|_{h}\|w\|_{V} .
$$

We now verify a variant of (2.7). Let $v, \phi \in V_{h}$ and $\psi=\phi-E_{h} \phi$. Then using (3.4) and (3.5), we get

$$
\begin{aligned}
(f, \psi)-a_{h}(v, \psi)= & (f, \psi)-\sum_{T \in \mathcal{T}_{h}} \int_{T} \nabla v \cdot \nabla \psi d x-\sum_{e \in \mathcal{E}_{h}} \frac{1}{h_{e}^{2}} \pi_{e}(\llbracket v \rrbracket) \pi_{e}(\llbracket \psi \rrbracket) \\
= & \left.\left.\sum_{T \in \mathcal{T}_{h}} \int_{T} f \psi d x-\sum_{e \in \mathcal{E}_{I}} \int_{e} \llbracket \nabla v \rrbracket \llbracket \psi\right\}\right\} d s \\
& -\sum_{e \in \mathcal{E}_{h}^{i}} \int_{e}\left\{\{\nabla v\} \pi_{e}(\llbracket \psi \rrbracket) d s+\sum_{e \in \mathcal{E}_{h}} \frac{1}{h_{e}^{2}} \pi_{e}(\llbracket u-v \rrbracket) \pi_{e}(\llbracket \psi \rrbracket)\right. \\
\leq & C\left(\|u-v\|_{h}+O s c_{1}(f)\right)\|\phi\|_{h}+C\left(\sum_{T \in \mathcal{T}_{h}} h_{T}^{2}\|\nabla u\|_{L_{2}(T)}^{2}\right)^{1 / 2}\|\phi\|_{h} .
\end{aligned}
$$

Therefore,

$$
\begin{aligned}
\sup _{\phi \in V_{h} \backslash\{0\}} \frac{\left(f, \phi-E_{h} \phi\right)-a_{h}\left(v, \phi-E_{h} \phi\right)}{\|\phi\|_{h}} \leq & C\left(\|u-v\|_{h}+O s c_{1}(f)\right) \\
& +C\left(\sum_{T \in \mathcal{T}_{h}} h_{T}^{2}\|\nabla u\|_{L_{2}(T)}^{2}\right)^{1 / 2} .
\end{aligned}
$$


Hence,

$$
\left\|u-u_{h}\right\|_{h} \leq C\left[\inf _{v \in V_{h}}\|u-v\|_{h}+O s c_{1}(f)+\left(\sum_{T \in \mathcal{T}_{h}} h_{T}^{2}\|\nabla u\|_{L_{2}(T)}^{2}\right)^{1 / 2}\right] .
$$

The error estimate in (3.3) is slightly different compared to the other DG methods. However, the estimate is still optimal up to the regularity of $u$.

Remark 3.3. We note that analogous error estimates hold for other DG formulations in [3, Table 3.2] and in [13].

\section{Fourth order PRoblems $(k=2)$}

The model problem is to find $u \in H_{0}^{2}(\Omega)$ such that

$$
a(u, v)=(f, v) \quad \forall v \in H_{0}^{2}(\Omega),
$$

where

$$
\begin{aligned}
a(w, v) & =\int_{\Omega} D^{2} w: D^{2} v d x \quad \forall w, v \in H_{0}^{2}(\Omega), \\
D^{2} w: D^{2} v & =\sum_{i, j=1}^{2} \frac{\partial^{2} w}{\partial x_{i} \partial x_{j}} \frac{\partial^{2} v}{\partial x_{i} \partial x_{j}} .
\end{aligned}
$$

Therefore, for this section $V=H_{0}^{2}(\Omega)$ and the norm $\|\cdot\|_{V}=|\cdot|_{H^{2}(\Omega)}$.

We define the Sobolev space $H^{s}\left(\Omega, \mathcal{T}_{h}\right)$ associated with the triangulation $\mathcal{T}_{h}$ as follows:

$$
H^{s}\left(\Omega, \mathcal{T}_{h}\right)=\left\{v \in L_{2}(\Omega): v_{T}=\left.v\right|_{T} \in H^{s}(T) \quad \forall T \in \mathcal{T}_{h}\right\} .
$$

For this section, we slightly alter the definition of the jumps and means.

For any $e \in \mathcal{E}_{h}^{i}$, there are two triangles $T_{+}$and $T_{-}$such that $e=\partial T_{+} \cap \partial T_{-}$. Let $n_{e}$ be the unit normal of $e$ pointing from $T_{-}$to $T_{+}$(cf. Fig. 3.1). For any $v \in H^{2}\left(\Omega, \mathcal{T}_{h}\right)$, we define the jump and mean of the normal derivative of $v$ on $e$ by

$$
\llbracket \frac{\partial v}{\partial n} \rrbracket=\left.\frac{\partial v_{+}}{\partial n_{e}}\right|_{e}-\left.\frac{\partial v_{-}}{\partial n_{e}}\right|_{e} \text { and } \quad\left\{\left\{\frac{\partial v}{\partial n}\right\}\right\}=\frac{1}{2}\left(\left.\frac{\partial v_{+}}{\partial n_{e}}\right|_{e}+\left.\frac{\partial v_{-}}{\partial n_{e}}\right|_{e}\right),
$$

where $v_{ \pm}=\left.v\right|_{T_{ \pm}}$.

Similarly, for any $v \in H^{3}\left(\Omega, \mathcal{T}_{h}\right)$, we define the jump and mean of the second order normal derivative across $e$ by

$$
\left.\llbracket \frac{\partial^{2} v}{\partial n^{2}} \rrbracket=\left.\frac{\partial^{2} v_{+}}{\partial n_{e}^{2}}\right|_{e}-\left.\frac{\partial^{2} v_{-}}{\partial n_{e}^{2}}\right|_{e} \text { and } \quad\left\{\frac{\partial^{2} v}{\partial n^{2}}\right\}\right\}=\frac{1}{2}\left(\left.\frac{\partial^{2} v_{+}}{\partial n_{e}^{2}}\right|_{e}+\left.\frac{\partial^{2} v_{-}}{\partial n_{e}^{2}}\right|_{e}\right) .
$$

For any edge $e \in \mathcal{E}_{h}^{b}$, there is a triangle $T \in \mathcal{T}_{h}$ such that $e=\partial T \cap \partial \Omega$. Let $n_{e}$ be the unit normal of $e$ that points outside $T$. For any $v \in H^{2}(T)$, we set

$$
\llbracket \frac{\partial v}{\partial n} \rrbracket=-\frac{\partial v_{T}}{\partial n_{e}},
$$

and for any $v \in H^{3}(T)$, we set

$$
\left\{\left\{\frac{\partial^{2} v}{\partial n^{2}}\right\}\right\}=\frac{\partial^{2} v_{T}}{\partial n_{e}^{2}}
$$

Let $V_{c} \subset H_{0}^{2}(\Omega)$ be the Hsieh-Clough-Tocher finite element space associated with $\mathcal{T}_{h}$ [19, 11. As in the previous section, the linear map $E_{h}: V_{h} \longrightarrow V_{c}$ is constructed 
by averaging [12, 16. Let $N$ be any (global) degree of freedom of $V_{c}$, i.e., $N$ is either the evaluation of a shape function or its first order derivatives at an interior node of $\mathcal{T}_{h}$, or the evaluation of the normal derivative of a shape function at a node on an interior edge. For $v_{h} \in V_{h}$, we define

$$
N\left(E_{h} v_{h}\right)=\frac{1}{\left|\mathcal{T}_{N}\right|} \sum_{T \in \mathcal{T}_{N}} N\left(v_{T}\right)
$$

where $\mathcal{T}_{N}$ is the set of triangles in $\mathcal{T}_{h}$ that share the degree of freedom, $N$ and $\left|\mathcal{T}_{N}\right|$ is the number of elements of $\mathcal{T}_{N}$.

We now derive the following discrete local efficiency estimates.

Lemma 4.1. Let $v \in V_{h}$. Then there is a positive constant $C$ independent of $h$ such that

$$
\begin{aligned}
& \sum_{T \in \mathcal{T}_{h}} h_{T}^{4}\left\|f-\Delta^{2} v\right\|_{L_{2}(T)}^{2} \leq C\left(\sum_{T \in \mathcal{T}_{h}}\left\|D^{2}(u-v)\right\|_{L_{2}(T)}^{2}+O s c_{2}(f)^{2}\right), \\
& \sum_{e \in \mathcal{E}_{h}^{i}} h_{e}\left\|\llbracket \partial^{2} v / \partial n^{2} \rrbracket\right\|_{L_{2}(e)}^{2} \leq C\left(\sum_{T \in \mathcal{T}_{h}}\left\|D^{2}(u-v)\right\|_{L_{2}(T)}^{2}+O s c_{2}(f)^{2}\right),
\end{aligned}
$$

where $\mathrm{Osc}_{2}(f)$ is defined in (2.8).

Proof. The proof is again based on bubble function techniques [16, 38].

Let $v \in V_{h}$ and $\bar{f} \in P_{r-2}(T)$ for an arbitrary $T \in \mathcal{T}_{h}$. Let $b_{T} \in P_{6}(T) \cap H_{0}^{2}(T)$ be the bubble function defined on $T$ such that $b_{T}\left(x_{T}\right)=1$, where $x_{T}$ is the barycenter of $T$. Let $\phi=b_{T}\left(\bar{f}-\Delta^{2} v\right)$ on $T$ and extend it to be zero on $\Omega \backslash T$. We have for some mesh independent constants $C_{1}$ and $C_{2}$ that

$$
C_{1}\left\|\bar{f}-\Delta^{2} v\right\|_{L_{2}(T)} \leq\|\phi\|_{L_{2}(T)} \leq C_{2}\left\|\bar{f}-\Delta^{2} v\right\|_{L_{2}(T)} .
$$

It follows from (4.1) and integration by parts that

$$
\begin{aligned}
& \left(f-\Delta^{2} v, \phi\right)=\int_{\Omega} f \phi d x-\int_{T} \Delta^{2} v \phi d x \\
& \quad=\int_{\Omega} D^{2} u: D^{2} \phi d x-\int_{T} D^{2} v: D^{2} \phi d x=\int_{T} D^{2}(u-v): D^{2} \phi d x .
\end{aligned}
$$

Using a standard inverse estimate [11, 19, we find

$$
\begin{aligned}
C_{1}\left\|\bar{f}-\Delta^{2} v\right\|_{L_{2}(T)}^{2} & \leq \int_{T}\left(\bar{f}-\Delta^{2} v\right) \phi d x=\int_{T}(\bar{f}-f) \phi d x+\int_{T}\left(f-\Delta^{2} v\right) \phi d x \\
& =\int_{T}(\bar{f}-f) \phi d x+\int_{T} D^{2}(u-v): D^{2} \phi d x \\
& \leq\|f-\bar{f}\|_{L_{2}(T)}\|\phi\|_{L_{2}(T)}+|u-v|_{H^{2}(T)}|\phi|_{H^{2}(T)} \\
& \leq\left(\|f-\bar{f}\|_{L_{2}(T)}+C h_{T}^{-2}|u-v|_{H^{2}(T)}\right)\|\phi\|_{L_{2}(T)} \\
& \leq C\left(\|f-\bar{f}\|_{L_{2}(T)}+h_{T}^{-2}|u-v|_{H^{2}(T)}\right)\left\|\bar{f}-\Delta^{2} v\right\|_{L_{2}(T)}
\end{aligned}
$$

which implies

$$
h_{T}^{2}\left\|\bar{f}-\Delta^{2} v\right\|_{L_{2}(T)} \leq C\left(|u-v|_{H^{2}(T)}+h_{T}^{2}\|f-\bar{f}\|_{L_{2}(T)}\right) .
$$

Using the triangle inequality, we obtain

$$
h_{T}^{2}\left\|f-\Delta^{2} v\right\|_{L_{2}(T)} \leq C\left(|u-v|_{H^{2}(T)}+h_{T}^{2}\|f-\bar{f}\|_{L_{2}(T)}\right)
$$

which implies (4.3). 
We now prove the second estimate (4.4). Let $e \in \mathcal{E}_{h}^{i}$ and $T_{ \pm}$be two triangles sharing the edge $e$. Let $\mathcal{T}_{e}$ be the set of the two triangles $T_{ \pm}$and let $n_{e}$ denote the unit normal of e pointing from $T_{-}$to $T_{+}$. (cf. Fig. 3.1).

Let $\beta$ be the jump $\llbracket \partial^{2} v / \partial n^{2} \rrbracket$ across $e$ and extend it outside $e$ so that it is constant along the lines perpendicular to $e$. Let $\zeta_{1} \in \mathbb{P}_{r-1}\left(T_{+} \cup T_{-}\right)$be defined by

$$
\zeta_{1}=0 \quad \text { on the edge } e \text { and } \quad \frac{\partial \zeta_{1}}{\partial n_{e}}=\beta
$$

A simple scaling argument shows that

$$
\begin{aligned}
\left|\zeta_{1}\right|_{H^{1}\left(T_{ \pm}\right)} & \approx\left(\int_{e} h_{e} \llbracket \llbracket \frac{\partial^{2} v}{\partial n^{2}} \rrbracket^{2} d s\right)^{1 / 2}, \\
\left\|\zeta_{1}\right\|_{L_{\infty}\left(T_{ \pm}\right)} & \approx\left(\int_{e} h_{e} \llbracket \frac{\partial^{2} v}{\partial n^{2}} \rrbracket^{2} d s\right)^{1 / 2} .
\end{aligned}
$$

Next we define $\zeta_{2} \in \mathbb{P}_{8}\left(T_{+} \cup T_{-}\right)$by the following properties:

(i) $\zeta_{2}$ vanishes to the first order on $\left(\partial T_{+} \cup \partial T_{-}\right) \backslash e$ (i.e., the union of the closed line segments $A P^{+}, A P^{-}, B P^{+}$and $B P^{-}$in Fig. 3.1).

(ii) $\zeta_{2}$ is positive on the (open) edge $e$.

(iii) $\int_{T_{+} \cup T_{-}} \zeta_{2} d x=\left|T_{+}\right|+\left|T_{-}\right|$.

By scaling we have

$$
\begin{aligned}
\int_{e} \zeta_{2} d s & \approx|e| \\
\left|\zeta_{2}\right|_{H^{1}\left(T_{ \pm}\right)} & \approx 1 \approx\left\|\zeta_{2}\right\|_{L_{\infty}\left(T_{ \pm}\right)} .
\end{aligned}
$$

It follows from (4.1), property (i) in the definition of $\zeta_{2}$, (4.5), (4.8), and integration by parts that

$$
\begin{aligned}
& C \int_{e} \llbracket \frac{\partial^{2} v}{\partial n^{2}} \rrbracket^{2} d s \leq \int_{e} \beta^{2} \zeta_{2} d s=\int_{e} \llbracket \frac{\partial^{2} v}{\partial n^{2}} \rrbracket \frac{\partial \zeta_{1}}{\partial n_{e}} \zeta_{2} d s=\int_{e} \llbracket \frac{\partial^{2} v}{\partial n^{2}} \rrbracket \frac{\partial\left(\zeta_{1} \zeta_{2}\right)}{\partial n_{e}} d s \\
&=-\sum_{T \in \mathcal{T}_{e}}\left(\int_{T} D^{2} v: D^{2}\left(\zeta_{1} \zeta_{2}\right) d x+\int_{T}\left(\nabla \cdot D^{2} v\right) \cdot \nabla\left(\zeta_{1} \zeta_{2}\right) d x\right) \\
&=-\sum_{T \in \mathcal{T}_{e}}\left(\int_{T} D^{2} v: D^{2}\left(\zeta_{1} \zeta_{2}\right) d x-\int_{T} \Delta^{2} v\left(\zeta_{1} \zeta_{2}\right) d x\right) \\
&= \sum_{T \in \mathcal{T}_{e}} \int_{T} D^{2}(u-v): D^{2}\left(\zeta_{1} \zeta_{2}\right) d x-\sum_{T \in \mathcal{T}_{e}} \int_{T} D^{2} u: D^{2}\left(\zeta_{1} \zeta_{2}\right) d x \\
& \quad+\sum_{T \in \mathcal{T}_{e}} \int_{T} \Delta^{2} v\left(\zeta_{1} \zeta_{2}\right) d x \\
&= \sum_{T \in \mathcal{T}_{e}} \int_{T} D^{2}(u-v): D^{2}\left(\zeta_{1} \zeta_{2}\right) d x-\sum_{T \in \mathcal{T}_{e}} \int_{T}\left(f-\Delta^{2} v\right)\left(\zeta_{1} \zeta_{2}\right) d x
\end{aligned}
$$

In view of the Poincaré inequality

$$
\left\|\zeta_{1} \zeta_{2}\right\|_{L_{2}\left(T_{ \pm}\right)} \leq C h_{T}^{2}\left|\zeta_{1} \zeta_{2}\right|_{H^{2}\left(T_{ \pm}\right)}
$$


and the inverse inequality

$$
\left|\zeta_{1} \zeta_{2}\right|_{H^{2}\left(T_{ \pm}\right)} \leq C h_{T}^{-1}\left|\zeta_{1} \zeta_{2}\right|_{H^{1}\left(T_{ \pm}\right)} \leq C h_{e}^{-1}\left|\zeta_{1} \zeta_{2}\right|_{H^{1}\left(T_{ \pm}\right)},
$$

we deduce from (4.10),

$$
\begin{aligned}
\int_{e} \llbracket \frac{\partial^{2} v}{\partial n^{2}} \rrbracket^{2} d s & \leq C \sum_{T \in \mathcal{T}_{e}}\left(|u-v|_{H^{2}(T)}+h_{T}^{2}\left\|f-\Delta^{2} v\right\|_{L_{2}(T)}\right)\left|\zeta_{1} \zeta_{2}\right|_{H^{2}(T)} \\
& \leq C \sum_{T \in \mathcal{T}_{e}}\left(|u-v|_{H^{2}(T)}+h_{T}^{2}\left\|f-\Delta^{2} v\right\|_{L_{2}(T)}\right) h_{e}^{-1}\left|\zeta_{1} \zeta_{2}\right|_{H^{1}\left(T_{ \pm}\right)} .
\end{aligned}
$$

From (4.6), (4.7) and (4.9) we have

$$
\begin{aligned}
\left|\zeta_{1} \zeta\right|_{H^{1}\left(T_{ \pm}\right)} & \leq\left|\zeta_{1}\right|_{L_{\infty}\left(T_{ \pm}\right)}\left|\zeta_{2}\right|_{H^{1}\left(T_{ \pm}\right)}+\left|\zeta_{1}\right|_{H^{1}\left(T_{ \pm}\right)}\left|\zeta_{2}\right|_{L_{\infty}\left(T_{ \pm}\right)} \\
& \leq C\left(\int_{e} h_{e} \llbracket \frac{\partial^{2} v}{\partial n^{2}} \rrbracket^{2} d s\right)^{1 / 2}
\end{aligned}
$$

which together with (4.11) implies

$$
\left(\int_{e} h_{e} \llbracket \frac{\partial^{2} v}{\partial n^{2}} \rrbracket^{2} d s\right)^{1 / 2} \leq C \sum_{T \in \mathcal{T}_{e}}\left(|u-v|_{H^{2}(T)}+h_{T}^{2}\left\|f-\Delta^{2} v\right\|_{L_{2}(T)}\right) .
$$

The estimate (4.4) now follows from (4.3) and (4.12).

We will now present the applications of Lemma 2.1 to a few discontinuous finite element methods for fourth order problems.

4.1. Morley nonconforming method. The discrete space [32] is

$$
\begin{aligned}
V_{h}=\left\{v \in L_{2}(\Omega):\right. & \left.v\right|_{T} \in P_{2}(T) \forall T \in \mathcal{T}_{h}, \\
& v \text { is continuous at all the vertices of } \mathcal{T}_{h}, \\
& \partial v / \partial n \text { is continuous at the midpoints of the edges of } \mathcal{T}_{h}, \\
& v=0 \text { at all the vertices on } \partial \Omega, \\
& \partial v / \partial n=0 \text { at all the midpoints of the edges on } \partial \Omega\} .
\end{aligned}
$$

The nonconforming method is to find $u_{h} \in V_{h}$ such that

$$
a_{h}\left(u_{h}, v\right)=(f, v) \quad \forall v \in V_{h},
$$

where for $w, v \in V_{h}$,

$$
a_{h}(w, v)=\sum_{T \in \mathcal{T}_{h}} \int_{T} D^{2} w: D^{2} v d x
$$

Note that $a_{h}$ is also well defined on $V_{c}$. Define the norm $\|v\|_{h}$ by $\|v\|_{h}^{2}=a_{h}(v, v)$. To verify (N2), let $v \in V, w \in V_{c}$ and $v_{h} \in V_{h}$. We then note that

$$
\left|a(v, w)-a_{h}\left(v_{h}, w\right)\right| \leq \sum_{T \in \mathcal{T}_{h}} \int_{T}\left|D^{2}\left(v-v_{h}\right)\right|\left|D^{2} w\right| d x \leq\left\|v-v_{h}\right\|_{h}\|w\|_{V} .
$$


The map $E_{h}$ defined in (4.2) satisfies the estimate [12, 16]

$$
\begin{aligned}
& \sum_{T \in \mathcal{T}_{h}}\left(h_{T}^{-4}\left\|v-E_{h} v\right\|_{L_{2}(T)}^{2}+h_{T}^{-2}\left|v-E_{h} v\right|_{H^{1}(T)}^{2}\right) \\
& +\left\|E_{h} v\right\|_{V}^{2} \leq C\|v\|_{h}^{2} \quad \forall v_{h} \in V_{h} .
\end{aligned}
$$

Let $v, \phi \in V_{h}$ and $\psi=\phi-E_{h} \phi$. For any $v \in V_{h}$ and for all vertices $p$ in $\mathcal{T}_{h}$, we have

$$
E_{h} v(p)=v(p)
$$

We find that

$$
\begin{aligned}
a_{h}\left(v, \phi-E_{h} \phi\right) & =\sum_{T \in \mathcal{T}_{h}} \int_{T} D^{2} v: D^{2}\left(\phi-E_{h} \phi\right) d x \\
& =\sum_{T \in \mathcal{T}_{h}} \int_{\partial T}\left(\frac{\partial^{2} v}{\partial n^{2}} \frac{\partial\left(\phi-E_{h} \phi\right)}{\partial n}+\frac{\partial^{2} v}{\partial \tau \partial n} \frac{\partial\left(\phi-E_{h} \phi\right)}{\partial \tau}\right) d s \\
& =-\sum_{e \in \mathcal{E}_{h}^{i}} \int_{e} \llbracket \frac{\partial^{2} v}{\partial n^{2}} \rrbracket\left\{\left\{\frac{\partial\left(\phi-E_{h} \phi\right)}{\partial n}\right\}\right\} d s .
\end{aligned}
$$

Here we have used the following consequence of (4.14):

$$
\sum_{T \in \mathcal{T}_{h}} \int_{\partial T} \frac{\partial^{2} v}{\partial \tau \partial n} \frac{\partial\left(\phi-E_{h} \phi\right)}{\partial \tau} d s=\sum_{T \in \mathcal{T}_{h}} \sum_{e \in \partial T} \frac{\partial^{2} v}{\partial \tau \partial n} \int_{e} \frac{\partial\left(\phi-E_{h} \phi\right)}{\partial \tau} d s=0
$$

where $\partial / \partial \tau$ denotes the tangential derivative along $\partial T$.

Using (4.3) and (4.4), we find that

$$
\begin{aligned}
(f, \psi)-a_{h}(v, \psi) & =(f, \psi)+\sum_{e \in \mathcal{E}_{h}^{i}} \int_{e} \llbracket \frac{\partial^{2} v}{\partial n^{2}} \rrbracket\left\{\left\{\frac{\partial \psi}{\partial n}\right\}\right\} d s \\
& \leq C\left(\sum_{T \in \mathcal{T}_{h}} h_{T}^{4}\|f\|_{L_{2}(T)}^{2}+\sum_{e \in \mathcal{E}_{h}^{i}} h_{e}\left\|\llbracket \partial^{2} v / \partial n^{2} \rrbracket\right\|_{L_{2}(e)}^{2}\right)^{1 / 2}\|\phi\|_{h} \\
& \leq C\left(\|u-v\|_{h}+\operatorname{Osc}_{2}(f)\right)\|\phi\|_{h} .
\end{aligned}
$$

Therefore,

$$
\sup _{\phi \in V_{h} \backslash\{0\}} \frac{\left(f, \phi-E_{h} \phi\right)-a_{h}\left(v, \phi-E_{h} \phi\right)}{\|\phi\|_{h}} \leq C\left(\|u-v\|_{h}+\operatorname{Osc}_{2}(f)\right) .
$$

Hence, the estimate (2.9) holds true.

4.2. $C^{0}$ interior penalty method. Define the space $V_{h}$ as

$$
V_{h}=\left\{v \in H_{0}^{1}(\Omega):\left.v\right|_{T} \in P_{2}(T) \forall T \in \mathcal{T}_{h}\right\}
$$

and the norm $\|\cdot\|_{h}$ on $V_{h}$ by

$$
\|v\|_{h}^{2}=\sum_{T \in \mathcal{T}_{h}} \int_{T} D^{2} v: D^{2} v d x+\sum_{e \in \mathcal{E}_{h}} \int_{e} \frac{\sigma}{h_{e}} \llbracket \partial v / \partial n \rrbracket^{2} d s \forall v \in V_{h},
$$

where $\sigma>0$ is the stabilizing parameter. 
The $C^{0}$ interior penalty method [12, 23] for (4.1) is to find $u_{h} \in V_{h}$ such that

$$
a_{h}\left(u_{h}, v\right)=(f, v) \quad \forall v \in V_{h},
$$

where for $w_{h}, v_{h} \in V_{h}$,

$$
\begin{aligned}
a_{h}\left(w_{h}, v_{h}\right)= & \sum_{T \in \mathcal{T}_{h}} \int_{T} D^{2} w_{h}: D^{2} v_{h} d x+\sum_{e \in \mathcal{E}_{h}} \int_{e}\left\{\left\{\frac{\partial^{2} w_{h}}{\partial n^{2}}\right\}\right\} \llbracket \frac{\partial v_{h}}{\partial n} \rrbracket d s \\
& +\sum_{e \in \mathcal{E}_{h}} \int_{e}\left\{\left\{\frac{\partial^{2} v_{h}}{\partial n^{2}}\right\}\right\} \llbracket \frac{\partial w_{h}}{\partial n} \rrbracket d s+\sum_{e \in \mathcal{E}_{h}} \frac{\sigma}{h_{e}} \int_{e} \llbracket \frac{\partial w_{h}}{\partial n} \rrbracket \llbracket \frac{\partial v_{h}}{\partial n} \rrbracket d s .
\end{aligned}
$$

Note that $a_{h}$ is well defined on $V_{c}$. The method is stable when $\sigma$ is sufficiently large. More precisely,

$$
a_{h}(v, v) \geq C\|v\|_{h}^{2} \quad \forall v \in V_{h}
$$

for $\sigma \geq \sigma_{*}>0$ and $\sigma_{*}$ sufficiently large.

The map $E_{h}$ defined in (4.2) satisfies the estimate [12, 16]

$$
\begin{gathered}
\sum_{T \in \mathcal{T}_{h}}\left(h_{T}^{-4}\left\|v-E_{h} v\right\|_{L_{2}(T)}^{2}+h_{T}^{-2}\left|v-E_{h} v\right|_{H^{1}(T)}^{2}\right) \\
+\left\|E_{h} v\right\|_{V}^{2} \leq C\|v\|_{h}^{2} \quad \forall v_{h} \in V_{h} .
\end{gathered}
$$

Let $v \in V, w \in V_{c}$ and $v_{h} \in V_{h}$. Then

$$
\begin{aligned}
a(v, w)-a_{h}\left(v_{h}, w\right) & =\sum_{T \in \mathcal{T}_{h}} \int_{T} D^{2}\left(v-v_{h}\right): D^{2} w d x+\sum_{e \in \mathcal{E}_{h}} \int_{e}\left\{\left\{\frac{\partial^{2} w}{\partial n^{2}}\right\}\right\} \llbracket \frac{\partial v_{h}}{\partial n} \rrbracket d s \\
& \leq C_{\sigma_{*}}\left\|v-v_{h}\right\|_{h}\|w\|_{V} .
\end{aligned}
$$

Again, using the fact that for any $v \in V_{h}, E_{h} v(p)=v(p)$ for all $v \in V_{h}$ and vertices $p$ in $\mathcal{T}_{h}$, we find the following analog of (4.15) for $v, \phi \in V_{h}$ and $\psi=\phi-E_{h} \phi$ :

$$
\begin{aligned}
\sum_{T \in \mathcal{T}_{h}} \int_{T} D^{2} v: D^{2} \psi d x=\sum_{T \in \mathcal{T}_{h}} \int_{\partial T}\left(\frac{\partial^{2} v}{\partial n^{2}} \frac{\partial \psi}{\partial n}+\frac{\partial^{2} v}{\partial \tau \partial n} \frac{\partial \psi}{\partial \tau}\right) d s \\
=-\sum_{e \in \mathcal{E}_{h}} \int_{e}\left\{\left\{\frac{\partial^{2} v}{\partial n^{2}}\right\}\right\} \llbracket \frac{\partial \psi}{\partial n} \rrbracket d s-\sum_{e \in \mathcal{E}_{h}^{i}} \int_{e}\left\{\left\{\frac{\partial \psi}{\partial n}\right\}\right\} \llbracket \frac{\partial^{2} v}{\partial n^{2}} \rrbracket d s
\end{aligned}
$$

Then using (4.18) and Lemma 4.1, we find

$$
\begin{aligned}
(f, \psi)- & \left.a_{h}(v, \psi)=(f, \psi)+\sum_{e \in \mathcal{E}_{h}^{i}} \int_{e} \llbracket \frac{\partial^{2} v}{\partial n^{2}} \rrbracket\left\{\frac{\partial \psi}{\partial n}\right\}\right\} d s \\
& -\sum_{e \in \mathcal{E}_{h}} \int_{e}\left\{\left\{\frac{\partial^{2} \psi}{\partial n^{2}}\right\}\right) \llbracket \frac{\partial v}{\partial n} \rrbracket d s-\sum_{e \in \mathcal{E}_{h}} \frac{\sigma}{h_{e}} \int_{e} \llbracket \frac{\partial v}{\partial n} \rrbracket \llbracket \llbracket \frac{\partial \psi}{\partial n} \rrbracket d s \\
\leq & C_{\sigma_{*}}\left(\|u-v\|_{h}+\operatorname{Osc}_{2}(f)\right)\|\phi\|_{h} .
\end{aligned}
$$

Hence, the assumption (2.7) is valid and the estimate (2.9) holds. 
4.3. Discontinuous Galerkin methods. The model problem (4.1) is rewritten in the following form. Find $u \in H_{0}^{2}(\Omega)$ such that

$$
a(u, v)=(f, v) \quad \forall v \in H_{0}^{2}(\Omega),
$$

where

$$
a(w, v)=\int_{\Omega} \Delta w \Delta v d x \forall w, v \in H_{0}^{2}(\Omega),
$$

and $\Delta$ denotes the Laplacian. Set $V=H_{0}^{2}(\Omega)$ and $\|v\|_{V}=|\Delta v|_{L^{2}(\Omega)}$ for all $v \in V$. For this last section, we switch back to the definitions of jump and mean in Section 3.

We now prove the following lemma.

Lemma 4.2. Let $v \in V_{h}$. Then there is a positive constant $C$ independent of $h$ such that

$$
\begin{gathered}
\sum_{T \in \mathcal{T}_{h}} h_{T}^{4}\left\|f-\Delta^{2} v\right\|_{L_{2}(T)}^{2} \leq C\left(\sum_{T \in \mathcal{T}_{h}}\|\Delta(u-v)\|_{L_{2}(T)}^{2}+O s c_{2}(f)^{2}\right), \\
\sum_{e \in \mathcal{E}_{h}^{i}} h_{e}\|\llbracket \Delta v \rrbracket\|_{L_{2}(e)}^{2} \leq C\left(\sum_{T \in \mathcal{T}_{h}}\|\Delta(u-v)\|_{L_{2}(T)}^{2}+O s c_{2}(f)^{2}\right),
\end{gathered}
$$

and

$$
\sum_{e \in \mathcal{E}_{h}^{i}} h_{e}^{3}\|\llbracket \nabla \Delta v \rrbracket\|_{L_{2}(e)}^{2} \leq C\left(\sum_{T \in \mathcal{T}_{h}}\|\Delta(u-v)\|_{L_{2}(T)}^{2}+O s c_{2}(f)^{2}\right),
$$

where $\mathrm{Osc}_{2}(f)$ is defined in (2.8).

Proof. The proofs of (4.20) and (4.21) are similar to the proofs of (4.3) and (4.4), respectively, and hence we omit the proofs.

We will prove (4.22) using the bubble function techniques [38, 16, 26]. Let $e \in \mathcal{E}_{h}^{i}$ and $T_{ \pm}$be the triangles sharing this edge $e$. Denote by $\mathcal{T}_{e}$ the patch of the two triangles $T_{ \pm}$(cf. Fig. 3.1). Consider $\llbracket \nabla \Delta v \rrbracket$ on $e$ and extend it to $T_{ \pm}$by constants along the lines orthogonal to $e$. Denote the resulting function $\zeta_{1} \in P_{r-3}\left(\mathcal{T}_{e}\right)$. It is then obvious that $\zeta_{1}=\llbracket \nabla \Delta v \rrbracket$ on $e$. Construct a piecewise polynomial bubble function $\zeta_{2} \in H_{0}^{2}\left(\mathcal{T}_{e}\right)$ such that $\zeta_{2}\left(x_{e}\right)=1$, where $x_{e}$ is the midpoint of $e$. Denote $\phi=\zeta_{1} \zeta_{2}$ and extend it to be zero on $\Omega \backslash \mathcal{T}_{e}$. We have by scaling [37,

$$
C\|\phi\|_{L^{2}\left(\mathcal{T}_{e}\right)} \leq\left(\int_{e} h_{e} \llbracket \nabla \Delta v \rrbracket^{2} d s\right)^{1 / 2}
$$


for some mesh independent constant $C$. Then, using (4.1), Poincaré's inequality and a standard inverse inequality, we have

$$
\begin{aligned}
\int_{e} \llbracket \nabla \Delta v \rrbracket^{2} d s \leq & C \int_{e} \llbracket \nabla \Delta v \rrbracket \zeta_{1} \zeta_{2} d s=C\left(\sum_{T \in \mathcal{T}_{e}} \int_{T}\left(\nabla \Delta v \cdot \nabla \phi+\Delta^{2} v \phi\right) d x\right) \\
= & \left.C\left(\sum_{T \in \mathcal{T}_{e}} \int_{T}\left(-\Delta v \Delta \phi+\Delta^{2} v \phi\right) d x\right)+C \sum_{T \in \mathcal{T}_{e}} \int_{\partial T} \llbracket \Delta v \rrbracket\{\nabla \phi\}\right] d s \\
= & C\left(\sum_{T \in \mathcal{T}_{e}} \int_{T} \Delta(u-v) \Delta \phi d x-\sum_{T \in \mathcal{T}_{e}} \int_{T}\left(f-\Delta^{2} v\right) \phi d x\right) \\
& +C \sum_{T \in \mathcal{T}_{e}} \int_{\partial T} \llbracket \Delta v \rrbracket\{\{\nabla \phi\} d s \\
\leq & C\left(\sum_{T \in \mathcal{T}_{e}}\left(h_{T}^{4}\left\|f-\Delta^{2} v\right\|_{L^{2}(T)}^{2}+|\Delta(u-v)|_{L^{2}(T)}^{2}\right)\right)^{1 / 2} h_{e}^{-2}\|\phi\|_{L^{2}\left(\mathcal{T}_{e}\right)} \\
& +C\left(\sum_{T \in \mathcal{T}_{e}} \int_{\partial T} h_{e} \llbracket \Delta v \rrbracket^{2} d s\right)^{1 / 2} h_{e}^{-2}\|\phi\|_{L^{2}\left(\mathcal{T}_{e}\right)}
\end{aligned}
$$

and then we use (4.20) and (4.21) to complete the proof of (4.22).

4.3.1. Symmetric interior penalty galerkin $(S I P G)$ method. Let

$$
V_{h}=\left\{v \in L_{2}(\Omega):\left.v\right|_{T} \in P_{r}(T) \forall T \in \mathcal{T}_{h}\right\}
$$

and define the norm $\|\cdot\|_{h}$ on $V_{h}$ by

$$
\|v\|_{h}^{2}=\sum_{T \in \mathcal{T}_{h}} \int_{T}|\Delta v|^{2} d x+\sum_{e \in \mathcal{E}_{h}} \int_{e} \frac{1}{h_{e}} \llbracket \nabla v \rrbracket^{2} d s+\sum_{e \in \mathcal{E}_{h}} \int_{e} \frac{1}{h_{e}^{3}} \llbracket v \rrbracket^{2} d s \quad \forall v \in V_{h} .
$$

The symmetric interior penalty method 4, 24, 33] is to find $u_{h} \in V_{h}$ such that

$$
a_{h}\left(u_{h}, v\right)=(f, v) \quad \forall v \in V_{h},
$$

where for $w, v \in V_{h}$,

$$
\begin{aligned}
a_{h}(w, v)= & \sum_{T \in \mathcal{T}_{h}} \int_{T} \Delta w \Delta v d x+\sum_{e \in \mathcal{E}_{h}} \int_{e}\{\{\nabla \Delta w\}\} \llbracket v \rrbracket d s-\sum_{e \in \mathcal{E}_{h}} \int_{e}\{\{\Delta w\}\} \llbracket \nabla v \rrbracket d s \\
& +\sum_{e \in \mathcal{E}_{h}} \int_{e}\left\{\{\nabla \Delta v\} \rrbracket \llbracket w \rrbracket d s-\sum_{e \in \mathcal{E}_{h}} \int_{e}\{\{\Delta v\}\} \llbracket \nabla w \rrbracket d s\right. \\
& +\sum_{e \in \mathcal{E}_{h}} \frac{\sigma_{1}}{h_{e}} \int_{e} \llbracket \nabla w \rrbracket \llbracket \nabla v \rrbracket d s+\sum_{e \in \mathcal{E}_{h}} \frac{\sigma_{2}}{h_{e}^{3}} \int_{e} \llbracket w \rrbracket \llbracket v \rrbracket d s
\end{aligned}
$$

and $\sigma_{1}>0, \sigma_{2}>0$ are penalty parameters.

For sufficiently large $\sigma_{1}$ and $\sigma_{2}$, it holds that

$$
a_{h}(v, v) \geq C\|v\|_{h}^{2} \quad \forall v \in V_{h},
$$


where $C$ is a mesh independent constant. Note for $v \in V, w \in V_{c}$ and $v_{h} \in V_{h}$ that

$$
\begin{aligned}
a(v, w)-a_{h}\left(v_{h}, w\right)= & \sum_{T \in \mathcal{T}_{h}} \int_{T} \Delta\left(v-v_{h}\right) \Delta w d x+\sum_{e \in \mathcal{E}_{h}} \int_{e}\{\{\nabla \Delta w\}\} \llbracket v-v_{h} \rrbracket d s \\
& -\sum_{e \in \mathcal{E}_{h}} \int_{e}\{\{\Delta w\}\} \llbracket \nabla\left(v-v_{h}\right) \rrbracket d s \\
\leq & C\left\|v-v_{h}\right\|_{h}\|w\|_{V} .
\end{aligned}
$$

The map $E_{h}$ defined in (4.2) satisfies the estimate [12, 16, 26]

$$
\begin{aligned}
& \sum_{T \in \mathcal{T}_{h}}\left(h_{T}^{-4}\left\|v-E_{h} v\right\|_{L_{2}(T)}^{2}+h_{T}^{-2}\left|v-E_{h} v\right|_{H^{1}(T)}^{2}\right) \\
& +\left\|E_{h} v\right\|_{V}^{2} \leq C\|v\|_{h}^{2} \quad \forall v_{h} \in V_{h} .
\end{aligned}
$$

Let $v, \phi \in V_{h}$ and $\psi=\phi-E_{h} \phi$. Then after two integration by parts and using earlier arguments with (4.20)-(4.22), we find that

$$
\begin{aligned}
(f, \psi)-a_{h}(v, \psi)= & \sum_{T \in \mathcal{T}_{h}} \int_{T}\left(f-\Delta^{2} v\right) \psi d x+\sum_{e \in \mathcal{E}_{h}} \int_{e} \llbracket \nabla \Delta v \rrbracket\{\{\psi\} d s \\
& -\sum_{e \in \mathcal{E}_{h}} \int_{e} \llbracket \Delta v \rrbracket\{\nabla \nabla \psi\} d s-\sum_{e \in \mathcal{E}_{h}} \int_{e}\{\{\nabla \Delta \psi\}\} \llbracket v \rrbracket d s \\
& +\sum_{e \in \mathcal{E}_{h}} \int_{e}\{\{\Delta \psi\}\} \llbracket \nabla v \rrbracket d s-\sum_{e \in \mathcal{E}_{h}} \frac{\sigma_{1}}{h_{e}} \int_{e} \llbracket \nabla v \rrbracket \llbracket \nabla \psi \rrbracket d s \\
& -\sum_{e \in \mathcal{E}_{h}} \frac{\sigma_{2}}{h_{e}^{3}} \int_{e} \llbracket v \rrbracket \llbracket \psi \rrbracket d s \\
\leq & C\left(\|u-v\|_{h}+\operatorname{Osc}_{2}(f)\right)\|\phi\|_{h} .
\end{aligned}
$$

Therefore, the assumption (2.7) is valid and hence the estimate (2.9) holds.

\section{Conclusions}

We have developed a new approach to discontinuous finite element methods and proved that all the well-known classical nonconforming methods and discontinuous Galerkin methods for second and fourth order elliptic problems are quasi-optimal up to higher order data oscillations, using only the weak formulations of the boundary value problems. Since the analysis involves techniques from a priori error analysis and a posteriori error analysis, it may be referred to as a medius error analysis.

This approach puts discontinuous finite element methods on an equal footing with conforming finite element methods in the sense that the first stage of the $a$ priori error analysis does not require the elliptic regularity theory. It is particularly useful for more complicated problems (such as interface problems) where the exact solution $u$ has low regularity, i.e., $u \in H^{s}(\Omega)$, where $s \leq 3 / 2$ for second order problems and $s \leq 7 / 2$ for fourth order problems.

To keep the technicalities to a minimum, the results in this paper are presented for simple model problems. With appropriate modifications these results can be extended to second order mixed boundary value problems, interface problems, nonhomogeneous Dirichlet problems and fourth order problems with different boundary conditions. 
The application of this new approach to $h p$ error estimates and nonconforming meshes will be investigated in the near future.

\section{ACKNOWLEDGMENTS}

The author would like to thank Susanne C. Brenner and Li-yeng Sung for many helpful suggestions.

\section{REFERENCES}

[1] M. Ainsworth and J. T. Oden. A posteriori error estimation in finite element analysis. Pure and Applied Mathematics (New York). Wiley-Interscience [John Wiley \& Sons], New York, 2000. MR1885308 (2003b:65001)

[2] D.N. Arnold. An interior penalty finite element method with discontinuous elements. SIAM J. Numer. Anal., 19:742-760, 1982. MR664882(83f:65173)

[3] D.N. Arnold, F. Brezzi, B. Cockburn and L.D. Marini. Unified analysis of discontinuous Galerkin methods for elliptic problems. SIAM J. Numer. Anal., 39:1749-1779, 2002. MR:1885715 (2002k:65183)

[4] G.A. Baker. Finite element methods for elliptic equations using nonconforming elements. Math. Comp., 31:45-59, 1977. MR.0431742 (55:4737)

[5] A. Berger, R. Scott and G. Strang. Approximate boundary conditions in the finite element method. In: Symposia Mathematica, Vol. X (Convegno di Analasi Numerica), London, Academic Press 1972, 295-313, 1972. MR0403258(53:7070)

[6] S.C. Brenner. Two-level additive Schwarz preconditioners for nonconforming finite element methods. Math. Comp., 65:897-921, 1996. MR1348039 (96j:65117)

[7] S.C. Brenner. Convergence of nonconforming multigrid methods without full elliptic regularity. Math. Comp., 68:25-53, 1999. MR.1620215 (99c:65229)

[8] S.C. Brenner. Ponicaré-Friedrichs inequalities for piecewise $H^{1}$ functions. SIAM J. Numer. Anal, 41:306-324, 2003. MR1974504 (2004d:65140)

[9] S.C. Brenner, K. Wang and J. Zhao. Poincaré-Friedrichs inequalities for piecewise $H^{2}$ functions. Numer. Funct. Anal. Optim., 25: 463-478, 2004. MR2106270 (2005i:65178)

[10] S.C. Brenner. Discrete Sobolev and Poincaré inequalities for piecewise polynomial functions. Elec. Trans. Numer. Anal., 18: 42-48, 2004. MR2083293(2005k:65239)

[11] S.C. Brenner and L.R. Scott. The Mathematical Theory of Finite Element Methods (Third Edition). Springer-Verlag, New York, 2008. MR2373954 (2008m:65001)

[12] S.C. Brenner and L.-Y. Sung. $C^{0}$ interior penalty methods for fourth order elliptic boundary value problems on polygonal domains. J. Sci. Comput., 22/23:83-118, 2005. MR2142191 (2005m:65258)

[13] S.C. Brenner and L. Owens. A weakly over-penalized non-symmetric interior penalty method. J. Numer. Anal. Indust. Appl. Math., 2: 35-48, 2007. MR2332345(2008c:65315)

[14] S.C. Brenner and L. Owens. A $W$-cycle algorithm for weakly over-penalized interior penalty method. Comput. Meth. Appl. Mech. engrg., 196: 3823-3832, 2007. MR2340007(2008i:65286)

[15] S.C. Brenner L. Owens and L.-Y. Sung. A weakly over-penalized symmetric interior penalty method. E. Tran. Numer. Anal, 30: 107-127, 2008. MR2480072 (2009k:65236)

[16] S.C. Brenner, T. Gudi and L.-Y. Sung. An a posteriori error estimator for a quadratic $C^{0}$ interior penalty method for the biharmonic problem. to appear in IMA J. Numer. Anal., doi:10.1093/imanum/drn057.

[17] P. Castillo, B. Cockburn, I. Perugia and D. Schötzau. An a priori error analysis of the local discontinuous Galerkin method for elliptic problems. SIAM J. Numer. Anal. , 38:1676-1706, 2000. MR1813251 (2002k:65175)

[18] C. Carstensen, T. Gudi and M. Jensen. A unifying theory of a posteriori error control for discontinuous Galerkin FEMs. Numer. Math., 112:363-379, 2009. MR2501309

[19] P.G. Ciarlet. The Finite Element Method for Elliptic Problems. North-Holland, Amsterdam, 1978. MR0520174 (58:25001)

[20] B. Cockburn and C.W. Shu. The local discontinuous Galerkin method for time-dependent convection-diffusion systems. SIAM J. Numer. Anal., 35:2440-2463, 1998. MR1655854 (99j:65163) 
[21] M. Crouzeix and P.A. Raviart. Conforming and Nonconforming finite element methods for solving the stationary Stokes equations. RAIRO 7, rev. 3:33-76, 1973. MR0343661 (49:8401)

[22] J. Douglas, Jr. and T. Dupont. Interior penalty procedures for elliptic and parabolic Galerkin methods. Lecture Notes in Phys., 58, Springer-Verlag, Berlin, 1976. MR0440955 (55:13823)

[23] G. Engel, K. Garikipati, T.J.R. Hughes, M.G. Larson, L. Mazzei, and R.L. Taylor. Continuous/discontinuous finite element approximations of fourth order elliptic problems in structural and continuum mechanics with applications to thin beams and plates, and strain gradient elasticity. Comput. Methods Appl. Mech. Engrg., 191:3669-3750, 2002. MR1915664 (2003d:74086)

[24] X. Feng and O.A. Karakashian. Fully discrete dynamic mesh discontinuous Galerkin methods for the Cahn-Hilliard equation in phase seperation. Math. Comp., 76:1093-1117, 2007. MR.2299767 (2008a:74048)

[25] X. Feng and O.A. Karakashian. Two-level nonoverlapping additive Schwarz methods for a discontinuous Galerkin approximation of the biharmonic problem. J. Sci. Comp., 22:299-324, 2005. MR 2142199 (2006b:65156)

[26] E. H. Georgoulis P. Houston, and J. Virtanen. An a posteriori error indicator for discontinuous Galerkin approximations of fourth order elliptic problems. Nottingham eprint.

[27] P. Grisvard. Elliptic Problems in Nonsmooth Domains. Pitman, Boston, 1985. MR775683 (86m:35044)

[28] T. Gudi, N. Nataraj and A. K. Pani. Mixed discontinuous Galerkin methods for the biharmonic equation. J. Sci. Comput., 37:103-232, 2008. MR2453216 (2009j:65319)

[29] P. Houston, D. Schötzau, and T.P. Wihler. Energy norm a posteriori error estimation of $h p$ adaptive discontinuous Galerkin methods for elliptic problems. Math. Models Methods Appl. Sci., pages 33-62, 2007. MR2290408 (2008a:65216)

[30] O.A. Karakashian and F. Pascal. A posteriori error estimates for a discontinuous Galerkin approximation of second-order elliptic problems. SIAM J. Numer. Anal., 41:2374-2399 (electronic), 2003. MR2034620 (2005d:65192)

[31] R.B. Kellogg. Singularities in interface problems, in: B. Hubbard (Ed.), Numerical Solutions of Partial Differential Equations II, Academic Press, New York, pp. 351-400, 1971. MR0289923 (44:7108)

[32] L.S.D. Morley. The triangular equilibrium problem in the solution of plate bending problems. Aero. Quart., 19:149-169, 1968.

[33] I. Mozolevski, E. Süli, and P.R. Bösing. $h p$-version a priori error analysis of interior penalty discontinuous Galerkin finite element approximations to the biharmonic equation. J. Sci. Comput., 30:465-491, 2007. MR2295480(2008c:65341)

[34] L. Owens. Multigrid Methods for Weakly Over-Penalized Interior penalty Methods. Ph.D. thesis, Department of Mathematics, University of South Corolina, 2007.

[35] S. Prudhomme, F. Pascal and J.T. Oden. Review of error estimation for discontinuous Galerkin methods. TICAM Report 00-27, October 17, 2000.

[36] B. Rivière, M.F. Wheeler, and V. Girault. A priori error estimates for finite element methods based on discontinuous approximation spaces for elliptic problems. SIAM J. Numer. Anal., 39:902-931, 2001. MR 1860450 (2002g:65149)

[37] R. Verfürth. A posteriori error estimation and adaptive mesh-refinement techniques. In Proceedings of the Fifth International Congress on Computational and Applied Mathematics (Leuven, 1992), volume 50, pages 67-83, 1994. MR1284252 (95c:65171)

[38] R. Verfürth. A Review of A Posteriori Error Estmation and Adaptive Mesh-Refinement Techniques. Wiley-Teubner, Chichester, 1995.

[39] M.F. Wheeler. An elliptic collocation-finite-element method with interior penalties. SIAM J. Numer. Anal., 15:152-161, 1978. MR0471383(57:11117)

Center for Computation and Technology, Louisiana State University, Baton Rouge, LOUISIANA 70803

E-mail address: tgudi@cct.1su.edu 